This item was submitted to Loughborough's Research Repository by the author.

Items in Figshare are protected by copyright, with all rights reserved, unless otherwise indicated.

\title{
Distributed closed-loop quasi-orthogonal space time block coding with four relay nodes: overcoming imperfect synchronization
}

PLEASE CITE THE PUBLISHED VERSION

PUBLISHER

(c) IEEE

VERSION

VoR (Version of Record)

\section{LICENCE}

CC BY-NC-ND 4.0

\section{REPOSITORY RECORD}

Elazreg, A.M., F.M. Abdurahman, and Jonathon Chambers. 2019. "Distributed Closed-loop Quasi-orthogonal Space Time Block Coding with Four Relay Nodes: Overcoming Imperfect Synchronization”. figshare. https://hdl.handle.net/2134/5549. 
This item was submitted to Loughborough's Institutional Repository (https://dspace.lboro.ac.uk/) by the author and is made available under the following Creative Commons Licence conditions.

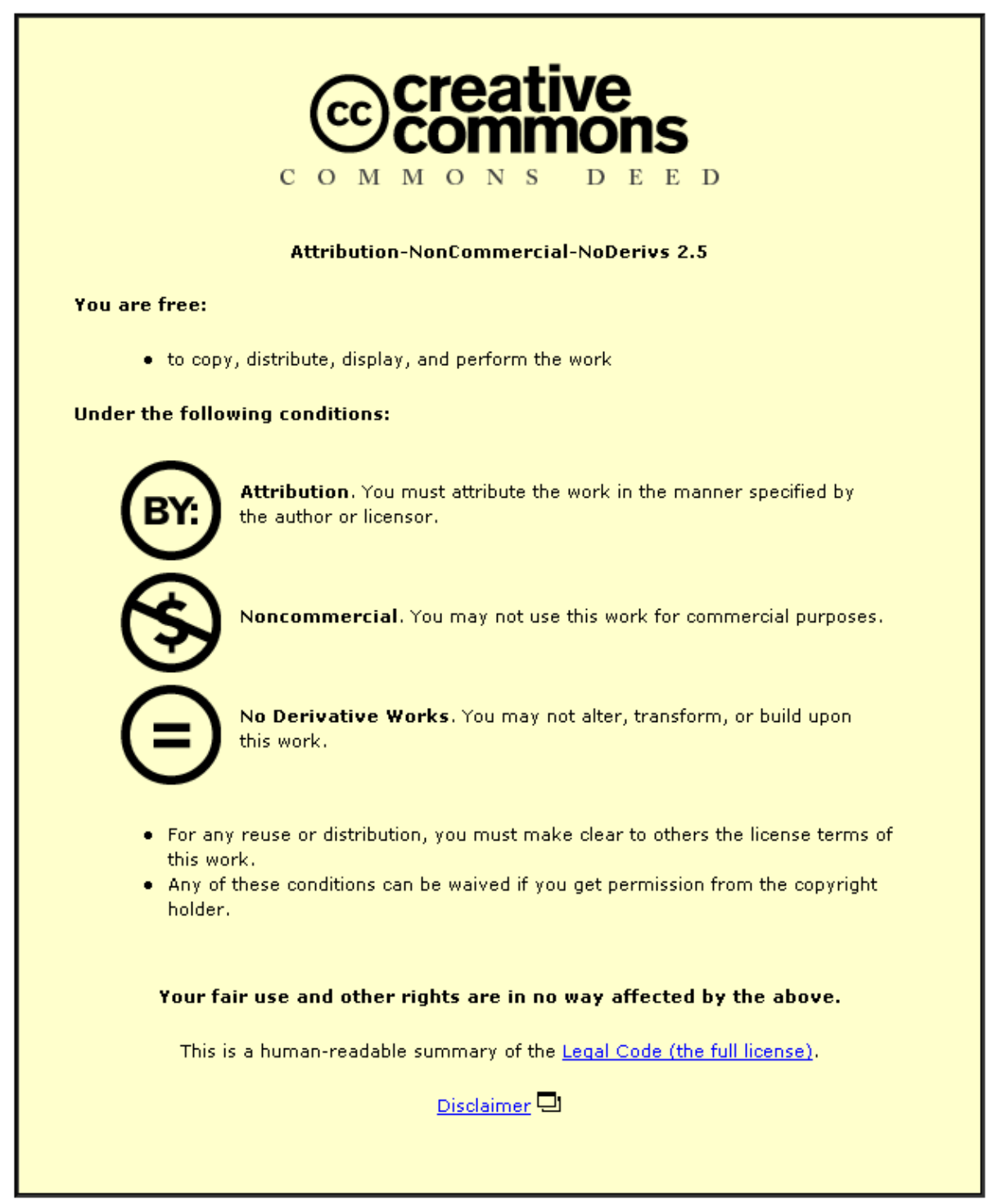

For the full text of this licence, please go to: http://creativecommons.org/licenses/by-nc-nd/2.5/ 


\title{
DISTRIBUTED CLOSED-LOOP QUASI-ORTHOGONAL SPACE TIME BLOCK CODING WITH FOUR RELAY NODES: OVERCOMING IMPERFECT SYNCHRONIZATION
}

\author{
A.M.Elazreg, F.M.Abdurahman, and J.A.Chambers \\ Advanced Signal Processing Group \\ Electronic and Electrical Engineering Department \\ Loughborough University, Leicestershire, UK,LE11 3TU \\ Email:\{ A.Elazreg, F.M.Abdurahman, J.A.Chambers\}@lboro.ac.uk \\ Tel: +44 (0)1509227029
}

\begin{abstract}
In this paper, closed-loop quasi-orthogonal space time block coding (QO-STBC) is exploited within a four relay node transmission scheme to achieve full-rate and increase the available diversity gain provided by earlier two relay approaches. The problem of imperfect synchronization between relay nodes is overcome by applying a parallel interference cancellation (PIC) detection scheme at the destination node. Bit error rate simulations confirm the advantages of the proposed methodology for a range of levels of imperfect synchronization and that only a small number of iterations is necessary within the PIC detection.
\end{abstract}

Index Terms - Closed-loop quasi-orthogonal space time block coding (CL QO-STBC), closed-loop phase rotated feedback, linear processing and parallel interference cancellation detection (PIC).

\section{INTRODUCTION}

Space time block coding (STBC) is a common techniques applied in multi-input multi-output (MIMO) wireless communication systems, which has proved to be a very effective technique to leverage spatial diversity and provide full data rate [1][2]. Owing to cost constraints, size limitation and hardware complexity, it is usually difficult to co-locate multiple antennas at one mobile communication node. It is, moreover, hard to achieve spatial uncorrelated in many point-to-point MIMO systems. However, STBC can be extended to cooperative systems [3], in the form of distributed space time block coding (D-STBC). By sharing antennas of different users, the reliability of transmission and full transmitter diversity can thereby be achieved [3][4][5].

The most widely used D-STBC strategies are focused on the case of two relay nodes using the Alamouti scheme [1], which provides full date rate and full diversity order, assuming that the cooperative relay nodes are perfectly synchronized at the symbol level, that means all transmitted symbols from all relay nodes arrive at their destination node at the same time. Practically, this is not a true representation of the real transmission environment. The assumption of accurate symbol level synchronization in wireless networks (such as ad hoc networks), is difficult or even impossible to achieve owing to the signalling overhead [6]. In the imperfect synchronization case between the relay nodes, the channel might become dispersive even under flat fading condition which will damage the orthogonality of the STBC and can lead to considerable performance impairment [11]. Previously, there has been limitated work reported regarding the mitigation of interference at the symbol level. The equalization technique was mainly used at the destination node to perform such mitigation [6][13]. Some other techniques were also used to overcome the problem of signal synchronization. One of which proposed a new STBC detection solution based on PIC detection [11][12]. In the case of four relay nodes under imperfect synchronization, which was considered in [12], the maximum symbol transmission rate by using a complex orthogonal space time block coding was 3/4 and the PIC detector had moderate computational complexity. However, in this paper, we propose a closed-loop QO-STBC under imperfect synchronization with linear processing detection with moderate computational complexity in the PIC detector building upon previous work in [12]. We show that the closed-loop QO-STBC can achieve full date rate (in the second phase of the network) and full diversity order with simple linear detection, contrary to what was previously achieved in [12].

The paper is organized as follows, Section II describes the system model of cooperative system protocol. In Section III the proposed closed-loop quasi-orthogonal space time block 
coding (QO-STBC) under imperfect synchronization is introduced. The full scheme of parallel interference cancelation (PIC) to mitigate the interference at the symbol level at the destination node is given in Section IV. Simulation results are shown in Section V. The final section summarizes this paper.

In the remaining part of this paper, [.] $]^{T},[.]^{*},||,. \Re\{$.$\} and$ $\{.\}^{H}$ denote "transpose", "conjugate","absolute value", "real part of complex number" and "Hermitian (complex conjugate transpose) of a matrix", respectively. $C N\left(0, \sigma^{2}\right)$ represent a Gaussian distributed complex number with the standard variance of $\sigma^{2}$ (i.e. $0.5 \sigma^{2}$ per dimension).

\section{SYSTEM MODEL}

In our model we consider a cooperative system with one source node (S), one destination node (D), and four relay nodes $\left(R_{m}\right)$, $m=1,2 . ., 4$ as depicted in Fig 1. Every node in the system



Fig. 1. Cooperative system architecture

has only one antenna, which generate virtual multiple antennas. To transmit the information from the $\mathrm{S}$ node to $\mathrm{D}$ node, the scheme comprises two phases. In the first phase, node $S$ transmits while node $\mathrm{D}$ and $R_{m}$ receive information. During the second phase, node $\mathrm{S}$ stops the transmission and the four relay nodes $R_{m}$ transmit the received signal after encoding to the node $\mathrm{D}$. The channel between any two nodes is assumed to be independent quasi-static flat Rayleigh fading. The channel gain from the $\mathrm{S}$ node to the $\mathrm{D}$ node is represented as $h_{s d}$, the channel gain from $\mathrm{S}$ node to $R_{m}$ node as $h_{s r_{m}}$, and the channel gain from $m$ th relay nodes to the D node as $h_{m}$.

\section{DISTRIBUTED CLOSED-LOOP QO-STBC UNDER IMPERFECT SYNCHRONIZATION}

The four relays mode closed-loop QO-STBC is depicted in Fig 2 and Fig 3, comprising the source node $S$, the destination node $\mathrm{D}$, the four relay nodes $\left(R_{1}, R_{2}, R_{3}, R_{4}\right)$ and the closed loop phase rotation feedback $U_{1}$ and $U_{2}$. Time division duplex transmission is assumed so that the channels can be assumed symmetric so that provision of channel state information (CSI) at the relays is feasible.
Phase 1: At the $\mathrm{S}$ node the data symbols are grouped into four symbols $s(i)=[s(1, i), s(2, i), s(3, i), s(4, i)]^{T}$ and then transmitted to the D node and the $R_{m}$ nodes as shown in Fig 2.

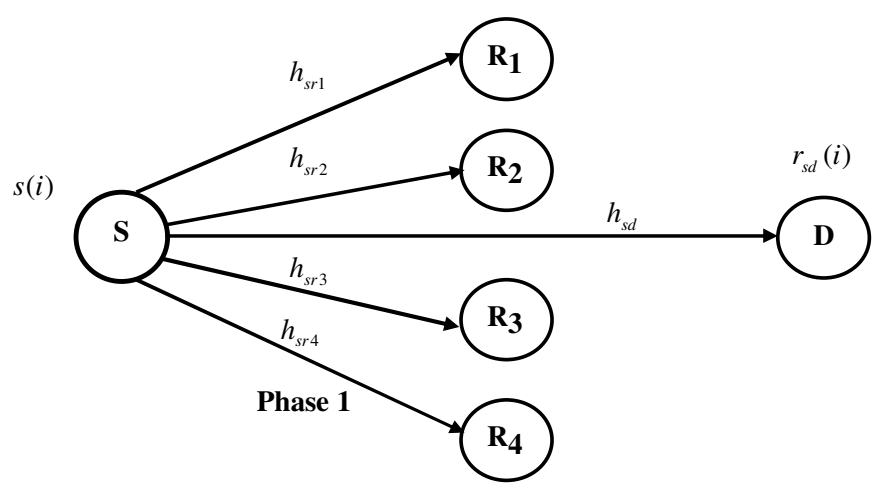

Fig. 2. Phase 1 relay mode with four relay nodes and a direct path

The received signal at the D node after passing through the channel gain $h_{s d}$ can be expressed as

$$
r_{s d}(i)=s(i) h_{s d}+n_{s d}(i)
$$

where $r_{s d}(i)=\left[r_{s d}(1, i), r_{s d}(2, i), r_{s d}(3, i), r_{s d}(4, i)\right]^{T}, h_{s d} \in$ $C N\left(0, \sigma^{2}\right)$ is the channel gain between $\mathrm{S}$ and $\mathrm{D}$ node, and $n_{s d}(i)=\left[n_{s d}(1, i), n_{s d}(2, i), n_{s d}(3, i), n_{s d}(4, i)\right]^{T}$, where $n_{s d}(j, i) \in C N\left(0, \sigma_{n}^{2}\right)$ is additive Gaussian noise.

The least squares (LS) method is used to detect which symbols reach the destination node $\mathrm{D}$.

$$
\hat{s}_{s d}(j, i)=\arg \left\{\left.\left.\min _{S_{l} \in S}\left|h_{s d}^{*} r_{s d}(j, i)-\right| h_{s d}\right|^{2} S_{l}\right|^{2}\right\}
$$

Phase 2: The received signals from the $\mathrm{S}$ node at $R_{m}$ are encoded as shown in Fig 3. The modulated signals from the third and fourth relays are respectively rotated by two phases, $\phi$ and $\theta$ to provide full fourth order diversity [7]. The relay mode of decode and forward is used in this paper. As such, a sufficient level of cyclic redundancy check (CRC) can be included into the data packet at $\mathrm{S}$ node, therefore the relaying will only happen if the data packet is correctly detected at $R_{m}$, this arrangement is termed "selective relaying" in [8]. The encoding data packet at $R_{m}$ corresponding to $s(i)$ is $x_{m}(i)=\left[x_{m}(1, i), x_{m}(2, i), x_{m}(3, i), x_{m}(4, i)\right]^{T}$. In [9], QOSTBC has been proposed with the following code in (3) to achieve full date rate for more than two antennas, which is transmitted by $R_{m}$, where $m=1, \ldots, 4$.

$$
\begin{aligned}
& {\left[\begin{array}{llll}
x_{1}(i) & x_{2}(i) & x_{3}(i) & x_{4}(i)
\end{array}\right]=} \\
& {\left[\begin{array}{rrrr}
s(1, i) & s(2, i) & s(3, i) & s(4, i) \\
-s^{*}(2, i) & s^{*}(1, i) & -s^{*}(4, i) & s^{*}(3, i) \\
-s^{*}(3, i) & -s^{*}(4, i) & s^{*}(1, i) & s^{*}(2, i) \\
s(4, i) & -s(3, i) & -s(2, i) & s(1, i)
\end{array}\right]}
\end{aligned}
$$






Fig. 3. Phase 2 relay mode with four relay nodes together with feedback scheme to achieve full diversity.

Note that since four symbols $x_{1}(j, i), x_{2}(j, i), x_{3}(j, i), x_{4}(j, i)$ are transmitted in four time slots, (3) presented a rate one code, where $j=1,2,3,4$ denotes time slot, as compare to work in [11] which achieves only $3 / 4$ data rate. In open-loop QO-STBC some of the nonzero off-diagonal terms appear in matrix $\mathbf{H}^{H} \mathbf{H}$ as shown in [7], which reduces the diversity gain of the code. As shown in Fig 3, the two feedback methods for QO-STBC are used to achieve full diversity and full code rate. The data packets transmitted from $R_{3}$ and $R_{4}$ are instead rotated by $U_{1}=e^{j \phi}$ and $U_{2}=e^{j \theta}$ respectively as shown in Fig 3, while the other two relays are kept unchanged. Due the imperfect synchronization, such as different propagation delays, the signals $x_{m}(i)$ will not generally arrive at the D node at the same time. Which means accurate synchronization is difficult or impossible to achieve[10]. As shown in Fig 4 there is normally a timing misalignment of $\tau_{m}$ among the received versions of these signals. At this point $\tau_{m}$ is assumed to be smaller than sample period $T$ [10], as shown in Fig 4. It will still cause intersymbol interference (ISI) from neighboring symbols at the D node, owing to sampling or matched filtering (whatever pulse shaping is used).



Fig. 4. Imperfect synchronization at the D node the received signal at D node is the superposition of four symbol
As shown in Fig 4, we assume that the received signal at the D node is perfectly synchronized to $R_{1},\left(\tau_{1}=0\right)$ therefore the received signals $r_{r d}(1, i), r_{r d}(2, i), r_{r d}(3, i)$ and $r_{r d}(4, i)$ at the $\mathrm{D}$ node in the four independent time intervals are expressed as follows

$$
\begin{aligned}
r_{r d}(1, i)= & \sum_{m=1}^{2} h_{m}(0) x_{m}(1, i)+\sum_{m=3}^{4} U_{m-2} h_{m}(0) \\
& x_{m}(1, i)+h_{2}(-1) x_{2}(4, i-1)+\sum_{m=3}^{4} U_{m-2} \\
& h_{m}(-1) x_{m}(4, i-1)+n_{r d}(1, i) \\
r_{r d}(k, i)= & \sum_{m=1}^{2} h_{m}(0) x_{m}(k, i)+\sum_{m=3}^{4} U_{m-2} h_{m}(0) \\
& x_{m}(k, i)+h_{2}(-1) x_{2}(k-1, i)+\sum_{m=3}^{4} U_{m-2} \\
& h_{m}(-1) x_{m}(k-1, i)+n_{r d}(k, i)
\end{aligned}
$$

where $k=2,3,4, n_{r d}(j, i) \in C N\left(0, \sigma_{n}^{2}\right)$ is additive Gaussian noise and $h_{m}(l)$ where $m=1,2,3,4$ are the channel gains between $R_{m}$ nodes and the $\mathrm{D}$ node under imperfect inter node synchronization which are assumed to be block Rayleigh fading from packet to packet, i.e $h_{m}(l) \in C N\left(0, \sigma_{r}^{2}\right)$ (note that $h_{1}(0)=h_{1}$ ). For fair comparison with a non-relay scheme, all relay nodes transmit at $1 / 4$ power i.e $\sigma_{r}^{2}=\sigma_{s}^{2} / 4$. Due to imperfect synchronization $h_{m}(-1)$ reflects the inter symbol interference from the previous symbol. The relative strength of $h_{m}(-1)$ will be expressed by ratio as follows [11][12].

$$
\beta_{m}=\left|h_{m}(-1)\right|^{2} /\left|h_{m}(0)\right|^{2}
$$

where $\beta_{m}$ is defined to reflect the composite impact of time delay $\tau_{m}$ and pulse shaping waveforms.

Similarly, equation $\left|h_{m}(-1)\right|^{2}+\left|h_{m}(0)\right|^{2} \leq\left|h_{m}\right|^{2}$ is used and we normally have $\beta_{m}=0$ for $\tau_{m}=0$, and $\beta_{m}=1$ (i.e. $0 \mathrm{~dB}$ ) for $\tau_{m}=0.5 T$. Substituting (3) into (4) and (5), the received signals at the $\mathrm{D}$ node are matched filtered ( and after complex conjugating the second and third symbols) over four independent time intervals are expressed in short notation as follows

$$
\mathbf{r}(i)=\mathbf{H} s(i)+\mathbf{I}(i)+\mathbf{n}_{r d}(i)
$$

where

$$
\begin{gathered}
\mathbf{r}(i)=\left[r_{r d}(1, i), r_{r d}^{*}(2, i), r_{r d}^{*}(3, i), r_{r d}(4, i)\right]^{T}, \\
\mathbf{H}=\left[\begin{array}{cccc}
h_{1}(0) & h_{2}(0) & U_{1} h_{3}(0) & U_{2} h_{4}(0) \\
h_{2}^{*}(0) & -h_{1}^{*}(0) & U_{2}^{*} h_{4}^{*}(0) & -U_{1}^{*} h_{3}^{*}(0) \\
U_{1}^{*} h_{3}^{*}(0) & U_{2}^{*} h_{4}^{*}(0) & -h_{1}^{*}(0) & -h_{2}^{*}(0) \\
U_{2} h_{4}(0) & -U_{1} h_{3}(0) & -h_{2}(0) & h_{1}(0)
\end{array}\right],
\end{gathered}
$$




$$
\mathbf{n}_{r d}(i)=\left[n_{r d}(1, i), n_{r d}^{*}(2, i), n_{r d}^{*}(1, i), n_{r d}(4, i)\right]^{T}
$$

and

$$
\mathbf{I}(i)=\left[I(1, i), I^{*}(2, i), I^{*}(3, i), I(4, i)\right]^{T}
$$

where

$$
\begin{aligned}
I(1, i)= & h_{2}(-1) x_{2}(4, i-1)+\sum_{m=3}^{4} U_{m-2} h_{m}(-1) \\
& x_{m}(4, i-1) \\
I(k, i)= & h_{2}(-1) x_{2}(k-1, i)+\sum_{m=3}^{4} U_{m-2} h_{m}(-1) \\
& x_{m}(k-1, i)
\end{aligned}
$$

$x_{m}(4, i-1)$ and $x_{m}(k-1, i)$ are defined according to (3). From (7), the conventional closed-loop QO-STBC detection can be carried out via the following standard two step procedure assuming perfect channel state information at the D node.

The detection vector $\mathbf{y}(i)=[y(1, i), y(2, i), y(3, i), y(4, i)]^{T}$ with the receive vector $\mathbf{r}(i)=\left[r_{r d}(1, i), r_{r d}^{*}(2, i), r_{r d}^{*}(3, i)\right.$ ,$\left.r_{r d}(4, i)\right]^{T}$ can be calculated

STEP 1: linear transform

$$
\begin{aligned}
\mathbf{y}(\mathbf{i}) & =[y(1, i), y(2, i), y(3, i), y(4, i)]^{T}=\mathbf{H}^{H} \mathbf{r}(i) \\
& =\Delta s(i)+\mathbf{H}^{H} \mathbf{I}(i)+\mathbf{v}(i)
\end{aligned}
$$

where $\mathbf{v}(i)=\mathbf{H}^{H} \mathbf{n}_{r d}(i)$ is a noise component. The matrix $\Delta=\mathbf{H}^{H} \mathbf{H}$ is a $4 \times 4$ matrix with entries $\gamma=\sum_{m=1}^{2}\left|h_{m}(0)\right|^{2}+$ $\sum_{m=3}^{4}\left|U_{m-2} h_{m}(0)\right|^{2}$ and $\alpha=\Re\left\{h_{1}^{*} h_{4} U_{2}-h_{2}^{*} h_{3} U_{1}\right\}$ as follows

$$
\Delta=\left[\begin{array}{llll}
\gamma & 0 & 0 & \alpha \\
0 & \gamma & -\alpha & 0 \\
0 & -\alpha & \gamma & 0 \\
\alpha & 0 & 0 & \gamma
\end{array}\right]
$$

Note that all the off-diagonal terms of $\Delta$ will be zero as in Alamouti's scheme [1], because the first and second terms of $\alpha$ are rotated by $e^{j \theta}$ and $e^{j \phi}$,so it is possible to make $\alpha$ zero. According to the above analysis, the phase rotated can be designed as shown in [7].

STEP 2: :Least-squares (LS) detection

$$
\hat{s}_{r d}(j, i)=\arg \left\{\min _{S_{m} \in S}\left|y(j, i)-\Delta S_{m}\right|^{2}\right\}
$$

The above procedure can suffer from significant detection error, because the $\mathbf{H}^{H} \mathbf{I}(i)$ component in (10) will damage the orthogonality of the closed-loop QO-STBC. However, this procedure can give good performance gain when the $h_{m}(-1)=$ 0 (i.e. $\tau_{m}=0$ in the case of perfect synchronization).

\section{PIC BASED DETECTION}

The principle of parallel interference cancelation is applied to remove the impact of imperfect synchronization of the interference component $\mathbf{I}(i)$ from (7), assuming perfect channel state information at the $\mathrm{D}$ node. If the interference component $\mathbf{I}(i)$ in (7) is removed, that operation the PIC detection would achieve maximum likelihood due to the closed-loop QO-STBC structure. Previously, PIC detection has been applied to a co-located STBC system [14]. Since $x_{m}(4, i-1)$ in (4) is already known if the detection process has been initialized properly, $I(1, i)$ can be removed during the initialization stage.

The PIC iteration process can then be used to mitigate the impact of $\mathbf{I}(i)$ in (7) as follows

\section{Initialization:}

1-set iteration number $\mathrm{K}=0$

2- From the received signal $\mathbf{r}(i)$ in (7) calculate

$$
\dot{\mathbf{r}}^{(0)}(i)=\left[\begin{array}{c}
\mathbf{r}_{r d}(1, i)-\mathbf{I}(1, i) \\
\mathbf{r}_{r d}^{*}(2, i) \\
\mathbf{r}_{r d}^{*}(3, i) \\
\mathbf{r}_{r d}(4, i)
\end{array}\right]
$$

3-Because of the poor performance of the conventional detector, the (DT) detection result in equation (2) has been used to initialize $s^{(K)}(i)$ :

$$
\begin{aligned}
s^{(0)}(i) & =\left[s^{(0)}(1, i), s^{(0)}(2, i), s^{(0)}(3, i), s^{(0)}(4, i)\right]^{T} \\
& =\left[\hat{s}_{s d}^{(0)}(1, i), \hat{s}_{s d}^{(0)}(2, i), \hat{s}_{s d}^{(0)}(3, i), \hat{s}_{s d}^{(0)}(4, i)\right]^{T}
\end{aligned}
$$

4-Set the iteration number $\mathrm{K}=1,2, \ldots . \mathrm{N}$

5 -Remove more ISI in (5) by calculating

$$
\dot{\mathbf{r}}^{(k)}(i)=\left[\begin{array}{c}
\mathbf{r}_{r d}(1, i)-\mathbf{I}(1, i) \\
\mathbf{r}_{r d}^{*}(2, i)-I^{*(K-1)}(2, i) \\
\mathbf{r}_{r d}^{*}(3, i)-I^{*(K-1)}(3, i) \\
\mathbf{r}_{r d}(4, i)-I^{(K-1)}(4, i)
\end{array}\right]
$$

where $I^{(K-1)}(m, i)$ is determined using $s^{(K-1)}(i)$ with $x_{m}^{(K-1)}(k-1, i)$ in (9)

6- Substitute $\mathbf{r}(i)$ in (10) with $\dot{\mathbf{r}}^{(K)}(i)$ to obtain $\mathbf{y}^{(K)}(i)$, and then apply the LS detection to $\mathbf{y}^{(K)}(i)$ to obtain the detection result of $\hat{\mathbf{s}}^{(K)}(j, i)$.

7-Repeat the process from point 4 until $K \leq N$

\section{SIMULATION RESULTS}

In this section, simulation results of our proposed closed-loop QO-STBC under imperfect synchronization is given. The BER performance against SNR was simulated by using an 8PSK gray mapping scheme as in [12], the decode and forward 
detection at $R_{m}$ is assumed, the perfect synchronization between $R_{1}$ and $\mathrm{D}$ node is assumed as well and the other relays node are not perfect synchronization to the $\mathrm{D}$ node, which cause ISI and all the simulations which have performed in this paper are uncoded. The signal to noise ratio (SNR) is defined as $S N R=\sigma_{s}^{2} / \sigma_{n}^{2}$ and all relay nodes transmit at $1 / 4$ power. The $\mathrm{x}$-axis represents the SNR, while the $\mathrm{y}$-axis represent the BER. A comparison was made between the BER performance of the proposed closed-loop QO-STBC with previous work in [12] under perfect synchronization as shown in Fig 5, which shows closed-loop QO-STBC achieves full data rate with fourth diversity order contrary to previous work. In Fig (5) and (6) the impact level of synchronization is shown by changing the value of $\beta_{m}$, that means the time delay between $R_{1}$ and the other relays is changed. Fig (5) shows the result of conventional detector under different $\beta_{m}$ value also the BER for DT, closed-loop QO-STBC and previous work in [12] under perfect synchronization are included as reference, Fig (5) shows the conventional detector is not effective to synchronization error even under small time misalignments $\beta_{m}=-6$. On the other hand, the PIC scheme is very effective to synchronization error even under large time misalignments $\beta_{m}=0$ as shown in Fig (6) when the number of PIC iterations $\mathrm{k}=3$. Fig (7) illustrates the BER of PIC iterations for $\mathrm{k}=0,1,2,3$ and $\beta_{m}=-5$, the figure shows the conventional detector dose not deliver the performances gain and even worse than the DT under imperfect synchronization, while the PIC scheme is very effective to mitigate the impact of imperfect synchronization, the second and third iteration deliver the performance gain.

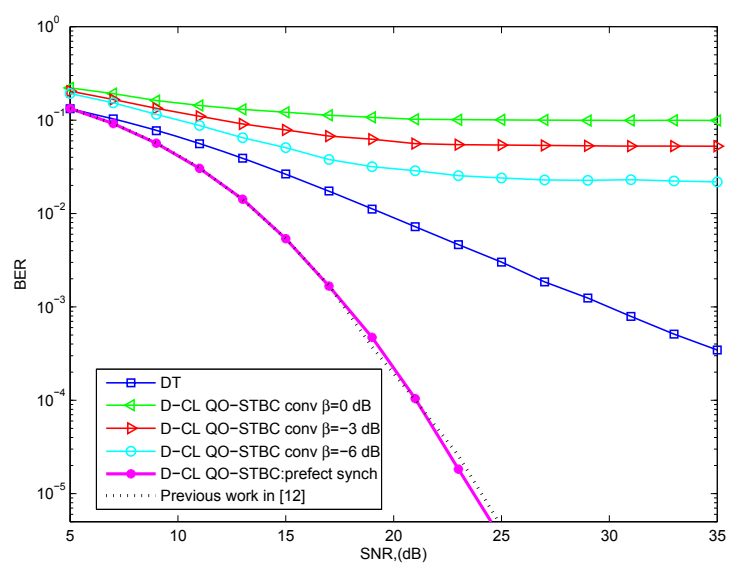

Fig. 5. The BER performance of conventional detection under different $\beta_{m}$ values

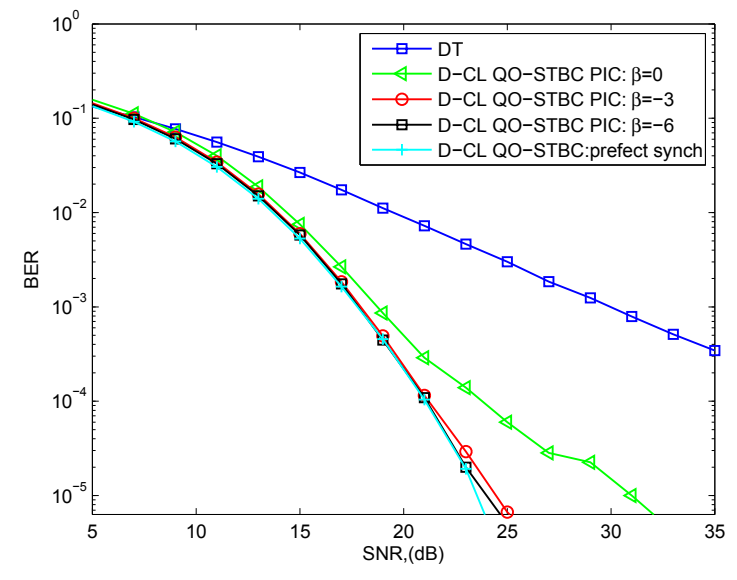

Fig. 6. The BER performance of PIC detection under different $\beta_{m}$ values

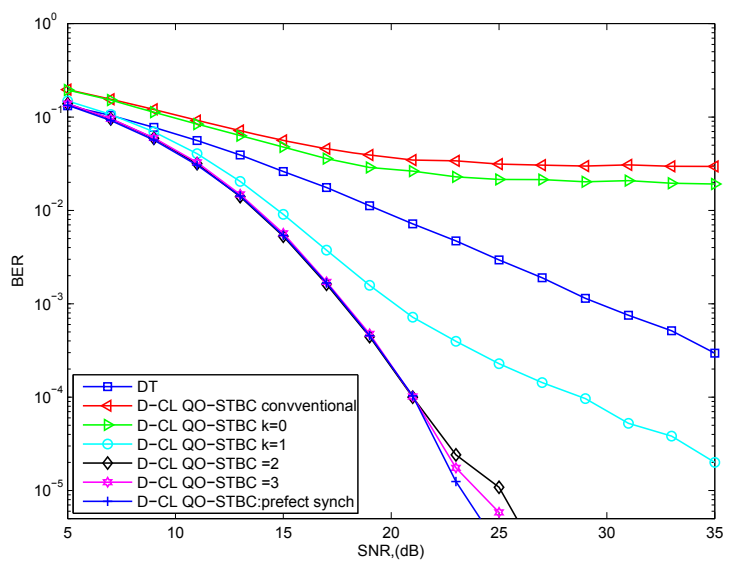

Fig. 7. The BER performance of PIC detection for different $K$ number of iterations

\section{CONCLUSION}

A distributed closed-loop QO-STBC transmission method with four relay nodes was investigated assuming imperfect synchronization. A PIC detection scheme was employed to mitigate the effect of interference on the system performance at the symbol level. A good performance with full date rate and simple linear detection is achieved by using closed-loop QO-STBC rather than previous work. Moreover, it has been shown that the conventional closed-loop QO-STBC detector is very sensitive to synchronization error and the PIC detector is very effective to mitigate the impact of imperfect synchronization. Finally the received signals during phase two can be combining with received signals during phase one (DT) to deliver significant performance gain. 


\section{REFERENCES}

[1] A.M. Alamouti, "A simple transmit diversity technique for wireless communications," IEEE J.Sel. Areas Commun., vol.16, no.8, pp.1451-1458, Oct 1998.

[2] V. Tarokh, H. Jafarkhani, and A. Calderbank, "Space time block codes from orthogonal design," IEEE Trans Inf. Theory, vol. 45, no. 5, pp.1456-1467, July 1999.

[3] J. Laneman, and G. Wornell, "Distributed Space-TimeCoded Protocols for Exploiting Cooperative Diversity in Wireless Networks," IEEE Trans. Inf. Theory, vol.49, no. 10, pp. 2415-2425, Oct 2003.

[4] A. Nosratinia, T.E Hunter,and A. Hedayat, " Cooperative communication in wireless networks," IEEE Communications Magazine, vol. 42, no. 10, pp. 74-80, Oct 2004.

[5] A. Sendonaris, E. Erkip, and B. Aazhang, "User cooperation diversity part I: System description," IEEE Trans. on Commun., vol. 51, no. 11, pp. 1927-1938, Nov. 2003.

[6] X. Li, "Space-time coded multi-transmission among distributed transmitters without perfect synchronization," IEEE Signal Processing Letters, vol. 11, no. 12, pp. 948-951,Dec 2004.

[7] C. Toker, S. Lambotharan, J. Chambers,"Closed-Loop quasi-orthogonal STBCs and their performance in multipath fading environments and when combined with turbo codes," IEEE Trans. on Wireless Commun., vol. 3, no. 6, pp.1890-1896, Nov. 2004.

[8] J. Laneman, D. Tse, and G. Wornell, "Cooperative diversity in wireless networks: efficient protocols and outage behaviour," IEEE Trans. Inf. Theory, vol. 50, no. 12, pp. 3062-3080, Dec 2004.

[9] H. Jafarkhani, “ A quasi-orthogonal space-time block code," IEEE Trans. Commun., vol. 49, no. 1, pp. 1-4, Jan 2001.

[10] S. Wei, D. Goeckel, and M. Valenti, "Asynchronous Cooperative Diversity," IEEE Trans. on Wireless Commu., vol. 5, no. 6, pp. 1547-1557, June 2006.

[11] F. Zheng, A. Burr, and S Olafsson, "PIC detector for distributed space-time block coding under imperfect synchronisation," IET Electronics Letters, vol. 43. no. 10, pp. 580-581, May 2007.

[12] F. Zheng, A. Burr, and S. Olafsson,"Distributed SpaceTime Block Coding for 3 and 4 Relay Nodes: Imperfect Synchronisation and a Solution,” The 18th Annual IEEE International Symposium PIMRC, Sept 2007.
[13] X. Li, F. Ng, J. Hwu, and M. Chen, “Channel equalization for STBC-encoded cooperative transmissions with asynchronous transmitters" Proc. of rhe 39th Asilomar conf. on Signsls Systems and Computer,pp.457-461, Oct 2005.

[14] F. Zheng, A. Burr, and S. Olafsson, “ Signal detection for orthogonal space time block coding over time selective fading channel: a Pic approach for the Gi system" IEEE Trans. Commu., vol. 53,no. 6, pp. 969-972, June 1998. 